\title{
THERMO-ELASTIC ANALYSIS OF A ROTATING HOLLOW CYLINDER MADE OF ARBITRARY FUNCTIONALLY GRADED MATERIALS*
}

\author{
Mohammad Arefi \\ Department of Solid Mechanic, Faculty of Mechanical Engineering, \\ University of Kashan, 87317-51167, Iran, \\ e-mail: arefi63@gmail.com, arefi@kashanu.ac.ir \\ IMAN NAHAS \\ School of Mechanical Engineering, Iran University of Science and Technology, \\ Narmak, Tehran, Iran, \\ e-mail: nahas@mecheng.iust.ac.ir \\ MAJID ABEDI \\ Department of Mechanical Engineering, \\ Shiraz University, Shiraz, Iran, \\ e-mail: majidabedi1989@gmail.com
}

[Received 18 May 2015. Accepted 16 November 2015]

\begin{abstract}
Thermo-mechanical analysis of the functionally graded orthotropic rotating hollow structures, subjected to thermo-mechanical loadings is studied in this paper. The relations were derived for both plane strain and plane stress conditions as a cylinder and disk, respectively. Non homogeneity was considered arbitrary through thickness direction for all mechanical and thermal properties. The responses of the system including temperature distribution, radial displacement and radial and circumferential stresses were derived in the general state. As case study, power law gradation was assumed for functionally graded cylinder and the mentioned results were evaluated in terms of parameters of the system such as non-homogeneous index and angular velocity.

KEY WORDS: Thermo-elastic, orthotropic, functionally graded materials, non-homogeneous.
\end{abstract}

\footnotetext{
${ }^{*}$ Corresponding author e-mail: arefi63@gmail.com

The corresponding author would like to gratefully acknowledge the financial support by University of Kashan (Grant Number 463865/08).
} 


\section{Introduction}

Changing different mechanical and material properties in terms of position is a requirement for different applications. This requirement has been responded using introduction of functionally graded materials by material scientist in Japan. Different variations of properties can be used by engineers to create best conditions of stress or displacement distribution. Directional properties such as orthotropic materials have benefits for loadings that in one direction are dominant rather than orthogonal directions. The present research tries to combine these two defined characteristics in the hollow cylinders.

Pan [1] derived exact solutions for three-dimensional, anisotropic, linearly elastic, and functionally graded rectangular composite laminates under simply supported edge conditions. The rectangular composite laminates were made of multilayered functionally graded material. Exponentially distribution along the thickness direction was considered for variable properties of layers. Response of a functionally graded thick cylindrical shell, subjected to thermal and mechanical loads was studied by Pelletier and Vel [2]. The cylindrical shell was assumed to be made of an orthotropic material. Results were presented for two-constituent isotropic and fiber-reinforced functionally graded shells. Exact solution of an infinitely long magneto elastic hollow cylinder and solid rotating cylinder polarized and magnetized radially, was presented by Babaei et al [3]. The cylinder was supposed orthotropic. Elastic problem of rotating functionally graded hollow polar orthotropic circular disks was investigated by Peng and Li [4]. They proposed Fredholm integral equation for arbitrary functionalities of material properties,. Two particular boundary conditions have been used for studying the numerical results.

Wang [5] presented elastic analysis of a rotating functionally graded hollow cylinder. The cylinder was divided to some sub cylinders and properties of every layer were considered constant. The radial and circumferential stresses along the radial direction were plotted for various values of non-homogeneity. The obtained results were validated with related references in a special case. Peng and Li [6] studied thermal stress analysis of a functionally graded hollow disk. The effect of gradient and temperature distribution was considered on the responses of the system. Allioglu et al [7] studied stress analysis of annular rotating discs made of functionally graded materials (FGMs). Elasticity modulus and density of the discs were assumed to vary radially, according to a power law function. Ootao and Ishihara [8] presented theoretical thermo-mechanical analysis of the functionally graded magneto-electro-elastic hollow sphere, due to uniform surface heating. They analyzed transient thermoelastic problem 
for a functionally graded hollow sphere, constructed of spherical isotropic and linear magneto-electro-thermoelastic materials using a laminated composite. Arefi and Rahimi [9] presented thermo-mechanical solution of a functionally graded cylinder with clamped-clamped boundary conditions, using first order shear deformation theory. They presented final differential equations in terms of displacement and rotation components. The coefficients of equations were defined in integral form. This form can be applicable for material with arbitrary functionality. Some useful equations and analysis of functionally graded materials can be considered in the related references [10-15].

This paper tries to develop thermo-elastic analysis of orthotropic hollow structures such as cylinder and disk made of functionally graded materials. Primary, fundamental relations are derived for an orthotropic material without any determined functionality. After these derivations, power law distribution is considered for studying an applied problem and investigation of numerical results of a cylinder

\section{Formulation of orthotropic hollow structure}

The basic relations for an orthotropic structure under three dimensional stress distributions are presented in this section. Hooke's law for an orthotropic structure by accounting thermal strains can be developed, as follows [6]:

$$
\left\{\begin{aligned}
\varepsilon_{r} & =\frac{1}{E_{r}(r)} \sigma_{r}-\frac{v_{\theta r}(r)}{E_{\theta}(r)} \sigma_{\theta}-\frac{v_{z r}(r)}{E_{z}(r)} \sigma_{z}+\alpha_{r}(r) T(r) \\
\varepsilon_{\theta} & =\frac{1}{E_{\theta}(r)} \sigma_{\theta}-\frac{v_{r \theta}(r)}{E_{r}(r)} \sigma_{r}-\frac{v_{z \theta}(r)}{E_{z}(r)} \sigma_{z}+\alpha_{\theta}(r) T(r) \\
\varepsilon_{z} & =\frac{1}{E_{z}(r)} \sigma_{z}-\frac{v_{\theta z}(r)}{E_{\theta}(r)} \sigma_{\theta}-\frac{v_{r z}(r)}{E_{r}(r)} \sigma_{r}+\alpha_{z}(r) T(r),
\end{aligned}\right.
$$

where $\varepsilon_{i}, \sigma_{i}(i=r, \theta, z)$ are strain and stress components, $v_{i j}(i \neq j, i, j=r$, $\theta, z)$ are Poisson ratio components, $E_{i}(i=r, \theta, z)$ are modulus of elasticity, $\alpha_{i}(i=r, \theta, z)$ is thermal expansion coefficient and $\mathrm{T}$ is temperature distribution. Mechanical properties such as Modulus of elasticity can be considered as functions of radial coordinate. For an orthotropic material, we have following additional relations for properties:

$$
\left\{\begin{array}{l}
\frac{v_{r \theta}(r)}{E_{r}(r)}=\frac{v_{\theta r}(r)}{E_{\theta}(r)} \\
\frac{v_{\theta z}(r)}{E_{\theta}(r)}=\frac{v_{z \theta}(r)}{E_{z}(r)} \\
\frac{v_{z r}(r)}{E_{z}(r)}=\frac{v_{r z}(r)}{E_{r}(r)} .
\end{array}\right.
$$




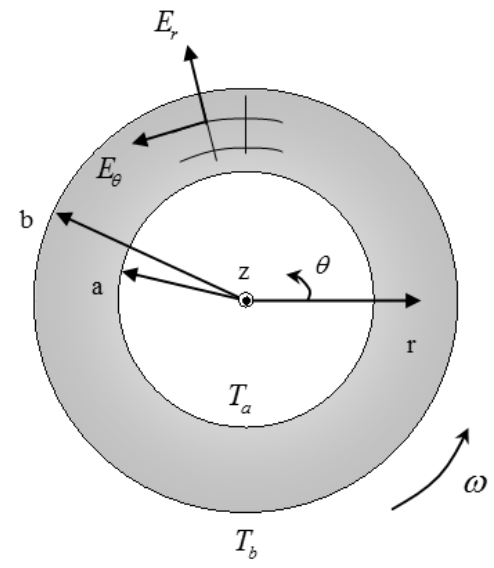

Fig. 1. The schematic figure of a rotating functionally graded orthotropic hollow cylinder

For guidance, the schematic figure of a rotating functionally graded hollow orthotropic structure is presented in Fig. 1. $E_{r}, E_{\theta}$, defined in Eq. (1), is modulus of elasticity in directions $r, \theta$ that is presented in Fig. 1.

These relations are due to symmetry of stiffness matrix. Considering Eq. (1), stress components can be evaluated in terms of strains and temperature distribution, as follows:

$$
\left\{\begin{aligned}
\sigma_{r}= & A_{1}(r) \varepsilon_{r}+A_{2}(r) \varepsilon_{\theta}-A_{1}(r) \alpha_{r}(r) T(r) \\
& -A_{2}(r) \alpha_{\theta}(r) T(r)-A_{4}(r) \alpha_{z}(r) T(r) \\
\sigma_{\theta}= & A_{2}(r) \varepsilon_{r}+A_{3}(r) \varepsilon_{\theta}-A_{2}(r) \alpha_{r}(r) T(r) \\
& -A_{3}(r) \alpha_{\theta}(r) T(r)-A_{5}(r) \alpha_{z}(r) T(r),
\end{aligned}\right.
$$

where, $A_{i}, i=1, \ldots, 5$ depend on the condition of plane strain or plane stress. By imposing the conditions of plane stress and strain for disk and cylinder structures, respectively, constants $A_{i}$ will be obtained. In Eq. (4), these constants are defined for disk as plane stress condition.

$$
\begin{aligned}
& A_{1}(r)=\frac{E_{r}(r) E_{\theta}(r)}{E_{\theta}(r)-E_{r}(r) v_{\theta r}^{2}(r)}, \\
& A_{2}(r)=\frac{E_{r}(r) E_{\theta}(r) v_{\theta r}(r)}{E_{\theta}(r)-E_{r}(r) v_{\theta r}^{2}(r)}, \\
& A_{3}(r)=\frac{E_{\theta}^{2}(r)}{E_{\theta}(r)-E_{r}(r) v_{\theta r}^{2}(r)}, \\
& A_{4}(r)=A_{5}(r)=0 .
\end{aligned}
$$


And for a cylinder with plane strain condition, we will have:

$$
\begin{aligned}
& A_{1}(r)=\frac{E_{r}^{2} E_{\theta}\left(E_{z}-E_{\theta} v_{z \theta}^{2}\right)}{E_{\theta}\left(E_{r}-E_{z} v_{r z}^{2}\right)\left(E_{z}-E_{\theta} v_{z \theta}^{2}\right)-E_{z}\left(E_{r} v_{\theta r}+E_{\theta} v_{r z} v_{z \theta}\right)^{2}} \\
& A_{2}(r)=\frac{E_{r} E_{\theta} E_{z}\left(E_{r} v_{\theta r}+E_{\theta} v_{r z} v_{z \theta}\right)}{E_{\theta}\left(E_{r}-E_{z} v_{r z}^{2}\right)\left(E_{z}-E_{\theta} v_{z \theta}^{2}\right)-E_{z}\left(E_{r} v_{\theta r}+E_{\theta} v_{r z} v_{z \theta}\right)^{2}} \\
& A_{3}(r)=\frac{E_{\theta}^{2} E_{z}\left(E_{r}-E_{z} v_{r z}^{2}\right)}{E_{\theta}\left(E_{r}-E_{z} v_{r z}^{2}\right)\left(E_{z}-E_{\theta} v_{z \theta}^{2}\right)-E_{z}\left(E_{r} v_{\theta r}+E_{\theta} v_{r z} v_{z \theta}\right)^{2}} \\
& A_{4}(r)=\frac{E_{r} E_{\theta} E_{z}\left[v_{r z}\left(E_{z}-E_{\theta} v_{z \theta}^{2}\right)+v_{z \theta}\left(E_{r} v_{\theta r}+E_{\theta} v_{r z} v_{z \theta}\right)\right]}{E_{\theta}\left(E_{r}-E_{z} v_{r z}^{2}\right)\left(E_{z}-E_{\theta} v_{z \theta}^{2}\right)-E_{z}\left(E_{r} v_{\theta r}+E_{\theta} v_{r z} v_{z \theta}\right)^{2}} \\
& A_{5}(r)=\frac{E_{\theta} E_{z}\left[E_{z} v_{r z}\left(E_{r} v_{\theta r}+E_{\theta} v_{r z} v_{z \theta}\right)+E_{\theta} v_{z \theta}\left(E_{r}-E_{z} v_{r z}^{2}\right)\right]}{E_{\theta}\left(E_{r}-E_{z} v_{r z}^{2}\right)\left(E_{z}-E_{\theta} v_{z \theta}^{2}\right)-E_{z}\left(E_{r} v_{\theta r}+E_{\theta} v_{r z} v_{z \theta}\right)^{2}} .
\end{aligned}
$$

Strain components, using a symmetric condition, can be derived as function of radial displacement $u_{r}$, as follows: $[5,6,10,15]$

$$
\varepsilon_{r}=\frac{d u_{r}}{d r}, \quad \varepsilon_{\theta}=\frac{u_{r}}{r}
$$

The stress components in Eq. (3) must satisfy equilibrium equations in presence of centrifugal force, as follows: $[5,6,10]$

$$
\frac{d \sigma_{r}}{d r}+\frac{\sigma_{r}-\sigma_{\theta}}{r}+\rho(r) r \omega^{2}=0 .
$$

In which, $\rho, \omega$ are density of used materials and angular velocity. By substitution of strain relations from Eq. (6) into first set of Eqs. (3), the radial displacement $u_{r}$ can be obtained from following equation:

(8) $u_{r}=$

$$
\frac{1}{\chi(r)}\left\{C_{1}+\int_{a}^{r} \frac{\chi(s)}{A_{1}(s)}\left[\begin{array}{l}
\sigma_{r}(s)+A_{1}(s) \alpha_{r}(s) T(s)+A_{2}(s) \alpha_{\theta}(s) T(s) \\
+A_{4}(s) \alpha_{z}(s) T(s)
\end{array}\right] d s\right\}
$$

where, $\chi(r)$ is an integral function of properties and radial coordinate, as follows:

$$
\chi(r)=\exp \left[\int_{a}^{r} \frac{A_{2}(s)}{s A_{1}(s)} d s\right]
$$


Using above definitions, circumferential stress can be derived as follows:

$$
\begin{aligned}
\sigma_{\theta}=\frac{A_{2}(r)}{A_{1}(r)} \sigma_{r}+\left[A_{3}(r)-\frac{A_{2}^{2}(r)}{A_{1}(r)}\right] & {\left[\frac{u_{r}}{r}-\alpha_{\theta}(r) T(r)\right] } \\
+ & {\left[\frac{A_{2}(r) A_{4}(r)}{A_{1}(r)}-A_{5}(r)\right] \alpha_{z}(r) T(r) . }
\end{aligned}
$$

Substitution of circumferential stress in equilibrium equation and solution of that yields radial stress, as follows:

$$
\begin{aligned}
& \frac{d \sigma_{r}}{d r}+\frac{1-\frac{A_{2}(r)}{A_{1}(r)}}{r} \sigma_{r}+\frac{\left[\frac{A_{2}^{2}(r)}{A_{1}(r)}-A_{3}(r)\right]}{r^{2} \chi(r)} \\
& \times \int_{a}^{r} \frac{\chi(s)}{A_{1}(s)}\left[\begin{array}{l}
\sigma_{r}(s)+A_{1}(s) \alpha_{r}(s) T(s) \\
+A_{2}(s) \alpha_{\theta}(s) T(s)+A_{4}(s) \alpha_{z}(s) T(s)
\end{array}\right] d s \\
&=C_{1} f(r)+f_{t}(r)-f_{\rho}(r),
\end{aligned}
$$

where, unknown functions are defined as in Eq. (12):

$$
\begin{aligned}
f(r)= & \frac{\left[A_{3}(r)-\frac{A_{2}^{2}(r)}{A_{1}(r)}\right]}{r^{2} \chi(r)}, \\
f_{t}(r)= & {\left[\frac{A_{2}^{2}(r)}{A_{1}(r)}-A_{3}(r)\right] \frac{\alpha_{\theta}(r) T(r)}{r} } \\
& \quad+\left[\frac{A_{2}(r) A_{4}(r)}{A_{1}(r)}-A_{5}(r)\right] \frac{\alpha_{z}(r) T(r)}{r}, \\
f_{\rho}(r)= & \rho(r) r \omega^{2} .
\end{aligned}
$$

Based on defined functions in Eq. (12) and integration of Eq. (11), relation containing radial stress can be defined as:

$$
\sigma_{r}+\int_{a}^{r} L(r, s) \sigma_{r}(s) d s=C_{1} F(r)+F_{t}(r)-F_{\rho}(r)+C_{2},
$$

where, three functions in right hand of Eq. (14) are defined:

$$
\left\{\begin{array}{l}
F(r)=\int_{a}^{r} f(s) d s, \\
F_{t}(r)=\int_{a}^{r} f_{t}(s) d s, \\
F_{\rho}(r)=\int_{a}^{r} f_{\rho}(s) d s
\end{array}\right.
$$


and two integration constants $\left(C_{1}, C_{2}\right)$, by imposing free stress condition at inner and outer radii $\left(\sigma_{r}(a)=\sigma_{r}(b)=0\right)$, are calculated as:

$$
\left\{\begin{array}{l}
C_{1}=\frac{F_{\rho}(b)-F_{t}(b)+\int_{a}^{b} L(b, s) \sigma_{r}(s) d s}{F(b)}, \\
C_{2}=0 .
\end{array}\right.
$$

\section{Heat conduction problem}

This section addresses thermal analysis of assumed structure. Due to symmetric condition, thermal distribution is a function of radial coordinate. Equation of heat transfer for a functionally graded material is [10, 11, 15]:

$$
\frac{1}{r} \frac{d}{d r}\left[r \lambda_{r}(r) \frac{d T(r)}{d r}\right]=0 .
$$

In which $\lambda_{r}$ is thermal conductivity coefficient along the radial direction. Similar to mechanical properties, thermal properties can be assumed as function of radial coordinate. Solution of Eq. (16) by two times integration with respect to $r$ yields temperature distribution in the integral form, as follows:

$$
T(r)=B_{1} \int_{a}^{r} \frac{1}{s \lambda_{r}(s)} d s+B_{2}
$$

where $B_{1}, B_{2}$ are constants of integration of Eq. (16). Imposing constant temperature at inner and outer radii $\left(T(a)=T_{a}, T(b)=T_{b}\right)$ presents temperature distribution, as follows:

$$
T(r)=\frac{T_{b}-T_{a}}{\int_{a}^{b} \frac{1}{s \lambda_{r}(s)} d s} \int_{a}^{r} \frac{1}{s \lambda_{r}(s)} d s+T_{a} .
$$

\section{Reduction of derived relations for a power law functionality}

Power law distribution of material properties can be considered in order to evaluate the results for an applied functionally graded material. For this functionality, we set all properties at outer radius and define gradation along the thickness direction, using a power function. For this functionality, we have variable properties, as follows [12-14]:

$$
\frac{E_{i}(r)}{E_{i}^{o}}=R^{\beta_{1}}, \quad \frac{\alpha_{i}(r)}{\alpha_{i}^{o}}=R^{\beta_{2}}, \quad \frac{\rho(r)}{\rho^{o}}=R^{\beta_{3}}, \quad \frac{\lambda_{r}(r)}{\lambda_{r}^{o}}=R^{\beta_{4}},
$$


where, $R=\frac{r}{b}$ is dimensionless variable through radial direction and $\beta_{i}, i=$ $1, \ldots, 4$ are four non-homogeneous indexes for different properties. Poisson ratios are considered to be constant. By defining the dimensionless radial displacement $\left(U_{r}=\frac{u_{r}}{b}\right)$, strain-displacement relations are defined as:

$$
\varepsilon_{r}=\frac{d u_{r}}{d r}=\frac{d U_{r}}{d R}, \quad \varepsilon_{\theta}=\frac{u_{r}}{r}=\frac{U_{r}}{R} .
$$

Substitution of strain-displacement relations from Eq. (20) and material properties from Eq. (19) into Eq. (3) presents radial and circumferential stress.

$$
\left\{\begin{array}{c}
\sigma_{r}=A_{1} R^{\beta_{1}} \frac{d U_{r}}{d R}+A_{2} R^{\beta_{1}-1} U_{r}-\left(A_{1} \alpha_{r}^{o}+A_{2} \alpha_{\theta}^{o}+A_{4} \alpha_{z}^{o}\right) R^{\beta_{1}+\beta_{2}} T(r) \\
\sigma_{\theta}=A_{2} R^{\beta_{1}} \frac{d U_{r}}{d R}+A_{3} R^{\beta_{1}-1} U_{r}-\left(A_{2} \alpha_{r}^{o}+A_{3} \alpha_{\theta}^{o}+A_{5} \alpha_{z}^{o}\right) R^{\beta_{1}+\beta_{2}} T(r),
\end{array}\right.
$$

where $A_{i}, i=1, \ldots, 5$ are constants, which can be evaluated form Eqs $(4,5)$ by changing $E_{i}, v_{i j}$ to $E_{i}^{o}, v_{i j}^{o}$. By setting $r=b R$, second side of Eq. 21 can be presented as function of $R$. Equilibrium equation in terms of dimensionless parameter of coordinate is:

$$
\frac{d \sigma_{r}}{d R}+\frac{\sigma_{r}-\sigma_{\theta}}{R}+\rho^{o} b^{2} \omega^{2} R^{\beta_{3}+1}=0 .
$$

Substitution of strain from Eq. (20) into Eq. (21) and then into Eq. (22) yields Euler equation, as follows:

$$
\frac{d^{2} U_{r}}{d R^{2}}+\left(\beta_{1}+1\right) \frac{1}{R} \frac{d U_{r}}{d R}+\frac{\beta_{1} A_{2}-A_{3}}{A_{1}} \frac{U_{r}}{R^{2}}=P(R) .
$$

In Eq. (23), $P(R)$ is non-homogeneous term including temperature distribution and centrifugal force, as follows:

$$
\begin{aligned}
P(R)=[( & \left(\beta_{1}+\beta_{2}+1\right)\left(\alpha_{r}^{o}+\frac{A_{2}}{A_{1}} \alpha_{\theta}^{o}+\frac{A_{4}}{A_{1}} \alpha_{z}^{o}\right) \\
& \left.-\left(\frac{A_{2}}{A_{1}} \alpha_{r}^{o}+\frac{A_{3}}{A_{1}} \alpha_{\theta}^{o}+\frac{A_{5}}{A_{1}} \alpha_{z}^{o}\right)\right] R^{\beta_{2}-1} T(b R) \\
& +\left(\alpha_{r}^{o}+\frac{A_{2}}{A_{1}} \alpha_{\theta}^{o}+\frac{A_{4}}{A_{1}} \alpha_{z}^{o}\right) R^{\beta_{2}} \frac{d T(b R)}{d R}-\frac{\rho^{o} b^{2} \omega^{2}}{A_{1}} R^{\beta_{3}-\beta_{1}+1}
\end{aligned}
$$


Solution of Eq. (23) is including homogeneous and particular solutions. Homogeneous solution is defined by calculation of roots of characteristic equation.

$$
\begin{aligned}
& U_{r}=\left(U_{r}\right)_{h}+\left(U_{r}\right)_{p}, \\
& \left(U_{r}\right)_{h}=C_{1} R^{m_{1}}+C_{2} R^{m_{2}} \quad m_{1,2}=-\frac{\beta_{1}}{2} \pm \sqrt{\frac{\beta_{1}^{2}}{4}-\frac{\beta_{1} A_{2}}{A_{1}}+\frac{A_{3}}{A_{1}}} .
\end{aligned}
$$

By employing the variable thermal properties in Eq. (19) and imposing constant temperature at inner and other radii, the radial distribution can be presented, as follows:

$$
T(R)=\frac{\left(T_{b}-T_{a}\right) k^{\beta_{4}} R^{-\beta_{4}}+k^{\beta_{4}} T_{a}-T_{b}}{k^{\beta_{4}}-1}
$$

where, $k=\frac{a}{b}$ and solution is valid for all values of $\beta_{4}$, except zero. Complete solution for $\beta_{4}=0$ can be developed in Appendix A. Substitution of temperature distribution from Eq. (26) into Eq. (24), determines particular solution, as follows:

$$
\left(U_{r}\right)_{p}=C_{3} R^{\beta_{2}-\beta_{4}+1}+C_{4} R^{\beta_{2}+1}+C_{5} R^{\beta_{3}-\beta_{1}+3} .
$$

Summation of particular and homogeneous solutions and substitution of result in stress-displacement relation (Eq. 21), tend to radial and circumferential stress distributions, as:

$$
\left\{\begin{aligned}
\sigma_{r}=C_{1}^{r} R^{\beta_{1}+m_{1}-1} & +C_{2}^{r} R^{\beta_{1}+m_{2}-1}+C_{3}^{r} R^{\beta_{1}+\beta_{2}-\beta_{4}} \\
& +C_{4}^{r} R^{\beta_{1}+\beta_{2}}+C_{5}^{r} R^{\beta_{3}+2} \\
\sigma_{\theta}=C_{1}^{\theta} R^{\beta_{1}+m_{1}-1} & +C_{2}^{\theta} R^{\beta_{1}+m_{2}-1}+C_{3}^{\theta} R^{\beta_{1}+\beta_{2}-\beta_{4}} \\
& +C_{4}^{\theta} R^{\beta_{1}+\beta_{2}}+C_{5}^{\theta} R^{\beta_{3}+2}
\end{aligned}\right.
$$

where $C_{i}^{r}, C_{i}^{\theta}, i=1, \ldots, 5$ are constants, which appears in Appendix B.

\section{Results and discussion}

The most important results for the cylinders made of functionally graded material obeying power law distribution, along the radial direction, can be evaluated in this section. The effect of two parameters can be investigated on the 
responses of the system. The first is angular velocity and the second is nonhomogeneous index. The other conditions such as temperature distribution and inner and outer pressures may be considered constants.

\subsection{The effect of angular velocities on the mechanical compo-} nents

In order to study the effect of angular velocity on the mechanical components, other boundary conditions are constrained as follows:

$$
\begin{aligned}
& \beta_{i}=1, \\
& \sigma_{r}(r=a)=\sigma_{r}(r=b)=0, \\
& T(r=a)=100, \quad T(r=b)=0 .
\end{aligned}
$$

The effect of different values of angular velocities can be considered on the radial distribution of mechanical components, such as radial, circumferential and Von-Mises stresses and radial displacement. Before presentation of the results, the dimensionless components must be assumed.

$$
U_{r}=\frac{u_{r}}{b}, \quad \bar{\sigma}_{r}=\frac{\sigma_{r}}{\rho b^{2} \omega^{2}}, \quad \bar{\sigma}_{\theta}=\frac{\sigma_{\theta}}{\rho b^{2} \omega^{2}}, \quad \bar{\sigma}_{M}=\frac{\sigma_{M}}{\rho b^{2} \omega^{2}}
$$

The radial distribution of dimensionless value of radial displacement in terms of different values of angular velocity is shown in Fig. 2. The obtained results indicate that the radial displacement increases with increasing angular velocity, considerably.

The radial distribution of radial, circumferential and Von-Mises stresses are presented in Figs 3, 4 and 5. We can find out that with increasing the angular velocity, the radial stress can change from compressive to tensile condition. Investigation on the circumferential stress indicates that this distribution approximately has linear manner. It can be observed, that for small values of angular velocity, the circumferential stress has an increasing behaviour unlike for large values of velocity, this behaviour is cancelled and almost circumferential stress approaches to a constant value. As we know, the Von Mises stress is function of radial and circumferential stresses. The numerical results of radial and circumferential stresses in Figs 3, 4 indicate, that the circumferential stress is much greater than radial stress. Therefore, the trend of Von Mises stress is in accordance with that one of circumferential stress, without considering the sign of circumferential stress. 


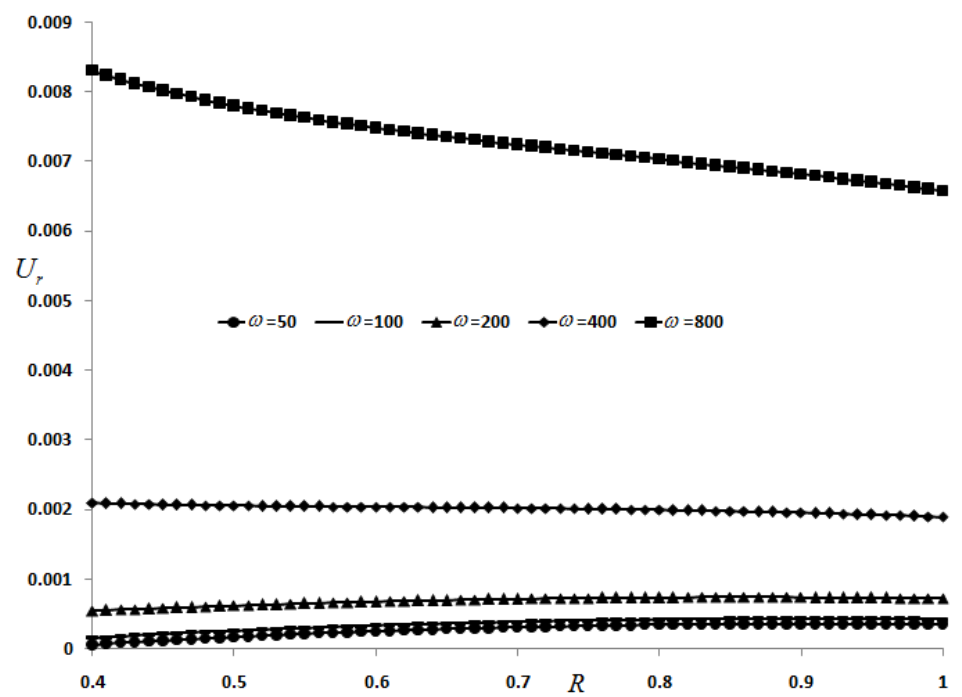

Fig. 2. The radial distribution of dimensionless radial displacement for different values of angular velocity

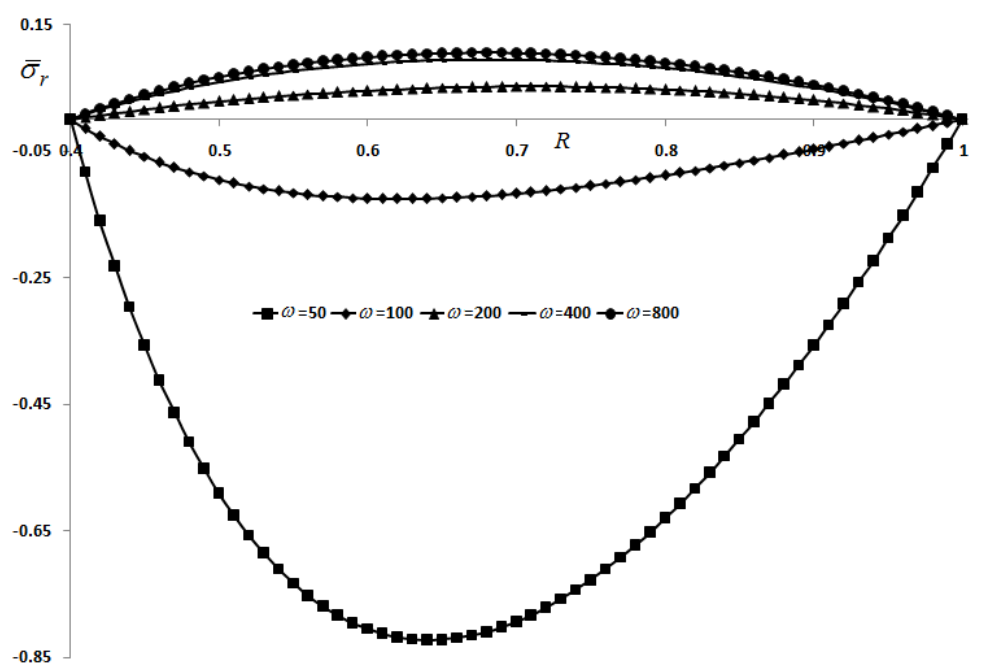

Fig. 3. The radial distribution of dimensionless radial stress for different values of angular velocity 


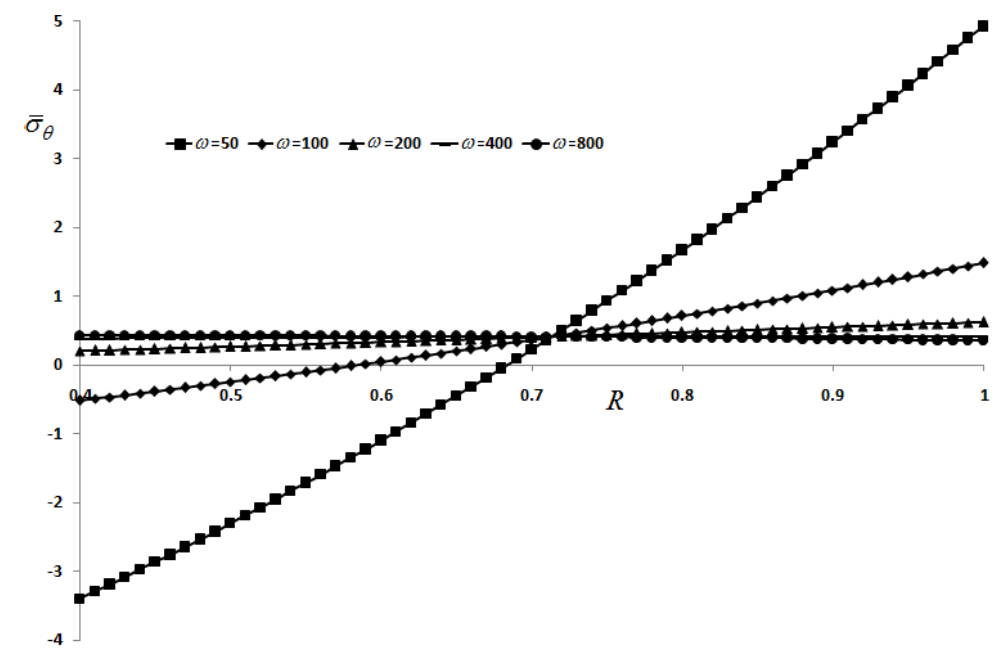

Fig. 4. The radial distribution of dimensionless circumferential stress for different values of angular velocity

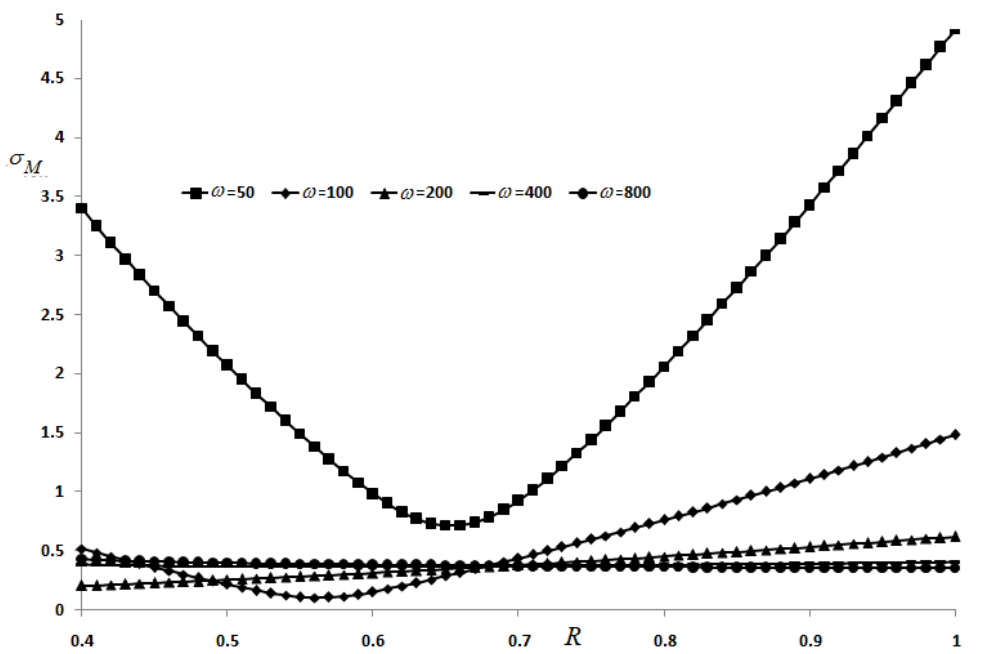

Fig. 5. The radial distribution of dimensionless Von-Mises stress for different values of angular velocity 


\subsection{The effect non-homogeneous index on the mechanical} components

This section evaluates the effect of non-homogeneous index on the stresses and displacement. Radial displacement, in terms of non-homogeneous index, is presented in Fig. 6. Angular velocity is considered 1000 for these evaluations.

The obtained results indicate that with increasing the non-homogeneous index, the radial displacement increases. This increasing is due to decreasing the modulus of elasticity, or stiffness of structure based on the defined functionality. As we scat from inner radius, the uniform manner of distribution is cancelled, because of anisotropic behaviour of material. The radial distribution of radial, circumferential and Von-Mises stresses are presented in Figs 7, 8 and 9 .

\section{Conclusion}

Thermo-elastic analysis of the functionally graded orthotropic rotating hollow structures was studied in this paper. The relations were derived for both plane strain and plane stress conditions. The general relations were reduced for a rotating functionally graded orthotropic cylinder. We can benefit from changing of gradient index to control displacement and stresses. It means, that for example, if you want to have a desired displacement, or stresses you can

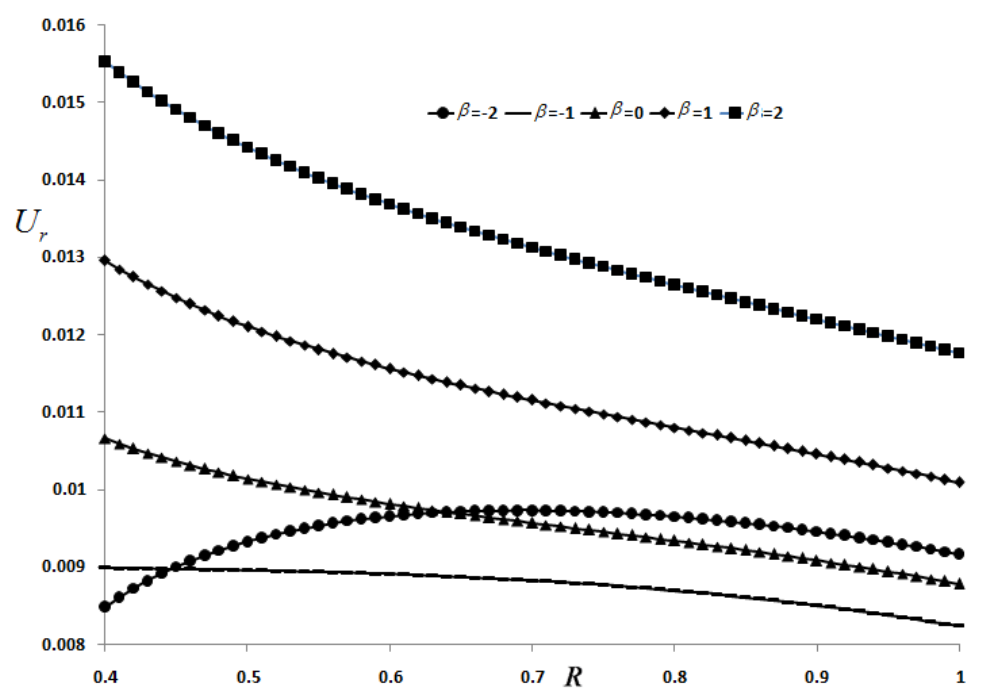

Fig. 6. The radial distribution of dimensionless radial displacement for different values of non-homogeneous index 


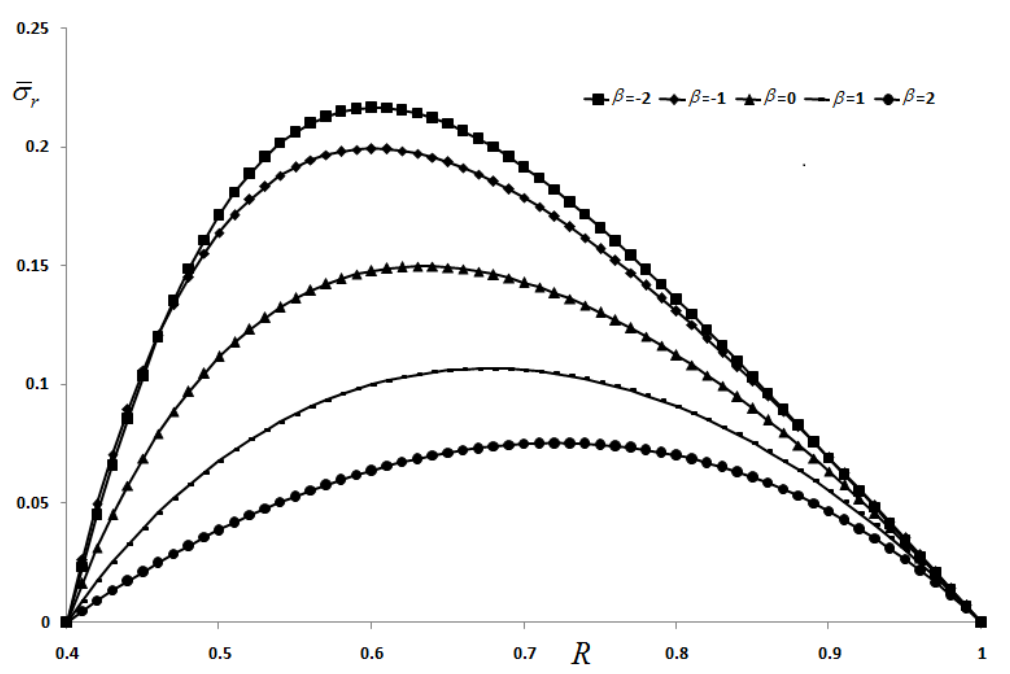

Fig. 7. The radial distribution of dimensionless radial stress for different values of non-homogeneous index

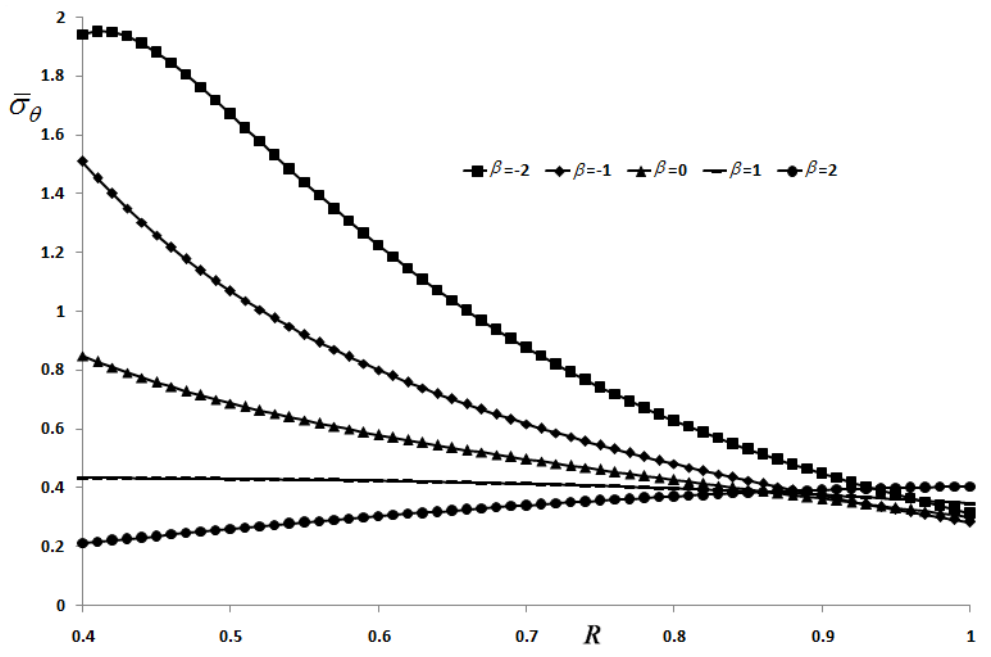

Fig. 8. The radial distribution of dimensionless circumferential stress for different values of non-homogeneous index 


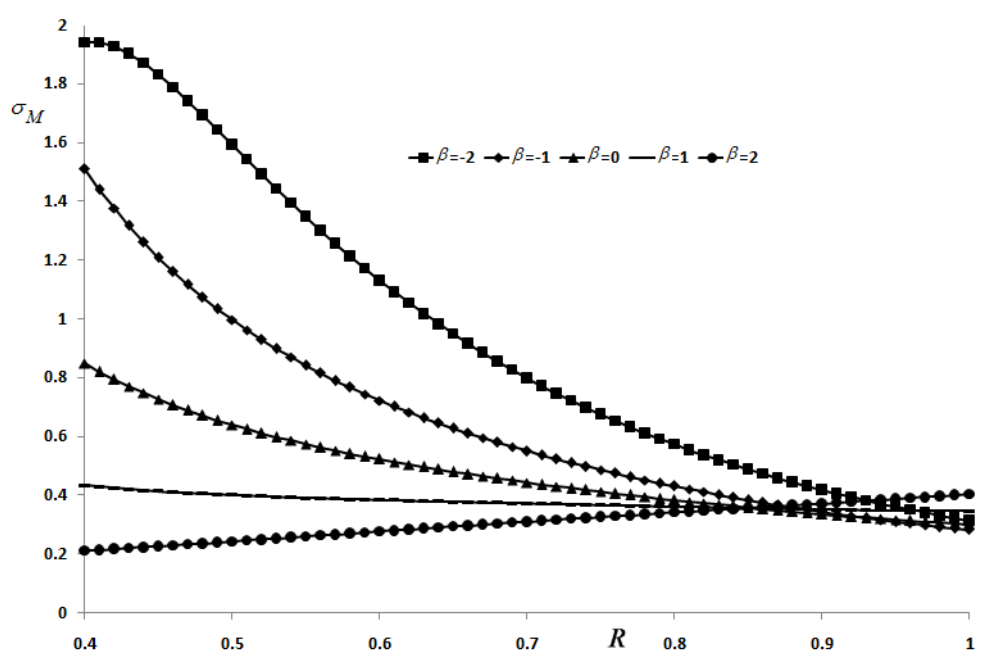

Fig. 9. The radial distribution of dimensionless Von-Mises stress for different values of non-homogeneous index

choose your optimal gradient index.

The most important conclusions can be regarded as:

1. In the first section of the paper, general solution for a hollow structure (cylinder or disk) made of functionally graded materials with centrifugal load was derived. The obtained solution can be applied for any functionality.

2. Increasing the angular velocity has increasing effect on the radial displacement of the orthotropic cylinder. Furthermore, this increasing tends to change the condition of radial stress from compressive to tensile.

3. Studying the effect of non homogeneous index on the radial displacement indicates that with increasing non-homogeneous index, radial displacement increases, due to decreasing the stiffness of the material. The circumferential stress is maximal at inner radial, because of applied thermal loading.

\section{Appendix A}

$$
\begin{aligned}
& T(r)=B_{1} \ln (R)+B_{2} . \\
& T(r)=B_{1} \ln (R)+B_{2} .
\end{aligned}
$$




$$
\left\{\begin{array}{l}
T(a)=T_{a}, \\
T(b)=T_{b} .
\end{array}\right.
$$

$$
\left\{\begin{array}{l}
B_{1}=\frac{T_{a}-T_{b}}{\ln (k)}, \\
B_{2}=T_{b} .
\end{array}\right.
$$

$$
T(r)=\frac{T_{a}-T_{b}}{\ln (k)} \ln (R)+T_{b} .
$$

$$
\left\{\begin{array}{c}
D_{1}=\frac{T_{a}-T_{b}}{\ln (k)}\left[\left(\beta_{1}+\beta_{2}+1\right)\left(\alpha_{r}^{o}+\frac{A_{2}}{A_{1}} \alpha_{\theta}^{o}+\frac{A_{4}}{A_{1}} \alpha_{z}^{o}\right)\right. \\
\left.\quad-\left(\frac{A_{2}}{A_{1}} \alpha_{r}^{o}+\frac{A_{3}}{A_{1}} \alpha_{\theta}^{o}+\frac{A_{5}}{A_{1}} \alpha_{z}^{o}\right)\right], \\
D_{2}=\frac{T_{a}-T_{b}}{\ln (k)}\left(\alpha_{r}^{o}+\frac{A_{2}}{A_{1}} \alpha_{\theta}^{o}+\frac{A_{4}}{A_{1}} \alpha_{z}^{o}\right) \\
\quad+T_{b}\left[\left(\beta_{1}+\beta_{2}+1\right)\left(\alpha_{r}^{o}+\frac{A_{2}}{A_{1}} \alpha_{\theta}^{o}+\frac{A_{4}}{A_{1}} \alpha_{z}^{o}\right)\right. \\
\left.\quad-\left(\frac{A_{2}}{A_{1}} \alpha_{r}^{o}+\frac{A_{3}}{A_{1}} \alpha_{\theta}^{o}+\frac{A_{5}}{A_{1}} \alpha_{z}^{o}\right)\right], \\
D_{3}=-\frac{\rho^{o} b^{2} \omega^{2}}{A_{1}} .
\end{array}\right.
$$

$$
\left\{\begin{aligned}
C_{3}= & \frac{D_{1}}{\left(\beta_{2}+1\right)\left(\beta_{1}+\beta_{2}+1\right)+\frac{\beta_{1} A_{2}-A_{3}}{A_{1}}} \\
C_{4}= & \frac{D_{2}}{\left(\beta_{2}+1\right)\left(\beta_{1}+\beta_{2}+1\right)+\frac{\beta_{1} A_{2}-A_{3}}{A_{1}}} \\
& -\frac{\left(\beta_{1}+2 \beta_{2}+2\right) D_{1}}{\left[\left(\beta_{2}+1\right)\left(\beta_{1}+\beta_{2}+1\right)+\frac{\beta_{1} A_{2}-A_{3}}{A_{1}}\right]^{2}} \\
C_{5}= & \frac{D_{3}}{\left(\beta_{3}+3\right)\left(\beta_{3}-\beta_{1}+3\right)+\frac{\beta_{1} A_{2}-A_{3}}{A_{1}}} .
\end{aligned}\right.
$$


Thermo-Elastic Analysis of a Rotating Hollow Cylinder ...

$U_{r}=C_{1} R^{m_{1}}+C_{2} R^{m_{2}}+C_{3} R^{\beta_{2}+1} \ln (R)+C_{4} R^{\beta_{2}+1}+C_{5} R^{\beta_{3}-\beta_{1}+3}$.

$(\mathrm{A}-10)$

$$
\left\{\begin{array}{r}
\sigma_{r}=C_{1}^{r} R^{\beta_{1}+m_{1}-1}+C_{2}^{r} R^{\beta_{1}+m_{2}-1} \\
\quad+C_{3}^{r} R^{\beta_{1}+\beta_{2}} \ln (R)+C_{4}^{r} R^{\beta_{1}+\beta_{2}}+C_{5}^{r} R^{\beta_{3}+2} \\
\sigma_{\theta}=C_{1}^{\theta} R^{\beta_{1}+m_{1}-1}+C_{2}^{\theta} R^{\beta_{1}+m_{2}-1} \\
\quad+C_{3}^{\theta} R^{\beta_{1}+\beta_{2}} \ln (R)+C_{4}^{\theta} R^{\beta_{1}+\beta_{2}}+C_{5}^{\theta} R^{\beta_{3}+2}
\end{array}\right.
$$

$$
\left\{\begin{array}{l}
C_{1}^{r}=\left(A_{1} m_{1}+A_{2}\right) C_{1}, \\
C_{2}^{r}=\left(A_{1} m_{2}+A_{2}\right) C_{2}, \\
C_{3}^{r}=\left[\left(\beta_{2}+1\right) A_{1}+A_{2}\right] C_{3}+\frac{\left(T_{b}-T_{a}\right)\left(A_{1} \alpha_{r}^{o}+A_{2} \alpha_{\theta}^{o}+A_{4} \alpha_{z}^{o}\right)}{\ln (k)}, \\
C_{4}^{r}=\left[\left(\beta_{2}+1\right) A_{1}+A_{2}\right] C_{4}+A_{1} C_{3}-T_{b}\left(A_{1} \alpha_{r}^{o}+A_{2} \alpha_{\theta}^{o}+A_{4} \alpha_{z}^{o}\right), \\
C_{5}^{r}=\left[\left(\beta_{3}-\beta_{1}+3\right) A_{1}+A_{2}\right] C_{5}
\end{array}\right.
$$

$$
\left\{\begin{array}{l}
C_{1}^{\theta}=\left(A_{2} m_{1}+A_{3}\right) C_{1}, \\
C_{2}^{\theta}=\left(A_{2} m_{2}+A_{3}\right) C_{2}, \\
C_{3}^{\theta}=\left[\left(\beta_{2}+1\right) A_{2}+A_{3}\right] C_{3}+\frac{\left(T_{b}-T_{a}\right)\left(A_{2} \alpha_{r}^{o}+A_{3} \alpha_{\theta}^{o}+A_{5} \alpha_{z}^{o}\right)}{\ln (k)}, \\
C_{4}^{\theta}=\left[\left(\beta_{2}+1\right) A_{2}+A_{3}\right] C_{4}+A_{2} C_{3}-T_{b}\left(A_{2} \alpha_{r}^{o}+A_{3} \alpha_{\theta}^{o}+A_{5} \alpha_{z}^{o}\right), \\
C_{5}^{\theta}=\left[\left(\beta_{3}-\beta_{1}+3\right) A_{2}+A_{3}\right] C_{5} .
\end{array}\right.
$$

$$
\left\{\begin{aligned}
C_{1}= & {\left[k^{\beta_{1}+\beta_{2}} \ln (k) C_{3}^{r}+\left(k^{\beta_{1}+\beta_{2}}-k^{\beta_{1}+m_{2}-1}\right) C_{4}^{r}\right.} \\
& \left.+\left(k^{\beta_{3}+2}-k^{\beta_{1}+m_{2}-1}\right) C_{5}^{r}\right] \\
& \times\left[\left(A_{1} m_{1}+A_{2}\right)\left(k^{\beta_{1}+m_{2}-1}-k^{\beta_{1}+m_{1}-1}\right)\right]^{-1} \\
C_{2}= & {\left[k^{\beta_{1}+\beta_{2}} \ln (k) C_{3}^{r}+\left(k^{\beta_{1}+\beta_{2}}-k^{\beta_{1}+m_{1}-1}\right) C_{4}^{r}\right.} \\
& \left.+\left(k^{\beta_{3}+2}-k^{\beta_{1}+m_{1}-1}\right) C_{5}^{r}\right] \\
& \times\left[\left(A_{1} m_{2}+A_{2}\right)\left(k^{\beta_{1}+m_{1}-1}-k^{\beta_{1}+m_{2}-1}\right)\right]^{-1}
\end{aligned}\right.
$$




\section{Appendix B}

(B-1)

$$
\left\{\begin{aligned}
C_{1}^{r}= & \left(A_{1} m_{1}+A_{2}\right) C_{1} \\
C_{2}^{r}= & \left(A_{1} m_{2}+A_{2}\right) C_{2}, \\
C_{3}^{r}= & {\left[\left(\beta_{2}-\beta_{4}+1\right) A_{1}+A_{2}\right] C_{3} } \\
& \quad+\frac{k^{\beta_{4}}\left(T_{a}-T_{b}\right)\left(A_{1} \alpha_{r}^{o}+A_{2} \alpha_{\theta}^{o}+A_{4} \alpha_{z}^{o}\right)}{k^{\beta_{4}}-1} \\
C_{4}^{r}= & {\left[\left(\beta_{2}+1\right) A_{1}+A_{2}\right] C_{4} } \\
& \quad+\frac{\left(T_{b}-k^{\beta_{4}} T_{a}\right)\left(A_{1} \alpha_{r}^{o}+A_{2} \alpha_{\theta}^{o}+A_{4} \alpha_{z}^{o}\right)}{k^{\beta_{4}}-1} \\
C_{5}^{r}= & {\left[\left(\beta_{3}-\beta_{1}+3\right) A_{1}+A_{2}\right] C_{5} . }
\end{aligned}\right.
$$$$
\left\{\begin{array}{l}
C_{1}^{\theta}=\left(A_{2} m_{1}+A_{3}\right) C_{1}, \\
C_{2}^{\theta}=\left(A_{2} m_{2}+A_{3}\right) C_{2}, \\
C_{3}^{\theta}=\left[\left(\beta_{2}-\beta_{4}+1\right) A_{2}+A_{3}\right] C_{3}
\end{array}\right.
$$

$$
\left\{\begin{array}{c}
+\frac{k^{\beta_{4}}\left(T_{a}-T_{b}\right)\left(A_{2} \alpha_{r}^{o}+A_{3} \alpha_{\theta}^{o}+A_{5} \alpha_{z}^{o}\right)}{k^{\beta_{4}}-1}, \\
C_{4}^{\theta}=\left[\left(\beta_{2}+1\right) A_{2}+A_{3}\right] C_{4} \\
+\frac{\left(T_{b}-k^{\beta_{4}} T_{a}\right)\left(A_{2} \alpha_{r}^{o}+A_{3} \alpha_{\theta}^{o}+A_{5} \alpha_{z}^{o}\right)}{k^{\beta_{4}}-1} \\
C_{5}^{\theta}=\left[\left(\beta_{3}-\beta_{1}+3\right) A_{2}+A_{3}\right] C_{5} .
\end{array}\right.
$$

$$
(\mathrm{B}-3)\left\{\begin{aligned}
C_{1}= & {\left[\left(k^{\beta_{1}+\beta_{2}-\beta_{4}}-k^{\beta_{1}+m_{2}-1}\right) C_{3}^{r}+\left(k^{\beta_{1}+\beta_{2}}-k^{\beta_{1}+m_{2}-1}\right) C_{4}^{r}\right.} \\
+ & \left.\left(k^{\beta_{3}+2}-k^{\beta_{1}+m_{2}-1}\right) C_{5}^{r}\right] \\
& \quad \times\left[\left(A_{1} m_{1}+A_{2}\right)\left(k^{\beta_{1}+m_{2}-1}-k^{\beta_{1}+m_{1}-1}\right)\right]^{-1}, \\
C_{2}= & {\left[\left(k^{\beta_{1}+\beta_{2}-\beta_{4}}-k^{\beta_{1}+m_{1}-1}\right) C_{3}^{r}+\left(k^{\beta_{1}+\beta_{2}}-k^{\beta_{1}+m_{1}-1}\right) C_{4}^{r}\right.} \\
+ & \left.\left(k^{\beta_{3}+2}-k^{\beta_{1}+m_{1}-1}\right) C_{5}^{r}\right] \\
& \times\left[\left(A_{1} m_{2}+A_{2}\right)\left(k^{\beta_{1}+m_{1}-1}-k^{\beta_{1}+m_{2}-1}\right)\right]^{-1} .
\end{aligned}\right.
$$




\section{REFERENCES}

[1] PAn, E. Exact Solution for Functionally Graded Anisotropic Elastic Composite Laminates. J. Compos. Mater., 37 (2003), No. 21, 1903-1920.

[2] Pelletier, J. L., S. S. Vel. An Exact Solution for the Steady-state Thermoelastic Response of Functionally Graded Orthotropic Cylindrical Shells. Int. J. Solids. Struct., 43 (2006), 1131-1158.

[3] Babaei, M. H., Z. T. Chen. Exact Solutions for Radially Polarized and Magnetized Magnetoelectroelastic Rotating Cylinders. Smart. Mater. Struct., 17 (2008), 025035, $11 \mathrm{pp}$.

[4] Peng, X. L. X. F. Li. Thermal Stress in Rotating Functionally Graded Hollow Circular Disks. Compos. Struct., 92 (2010), 1896-1904.

[5] Wang, H. M. Effect of Material Inhomogeneity on the Rotating Functionally of a Graded Orthotropic Hollow Cylinder. J. Mech. Sci. Tech., 24 (2010), No. 9, 1839-1844.

[6] Peng, X. L., X. F. Li. Elastic Analysis of Rotating Functionally Graded Polar Orthotropic Disks. Int. J. Mech. Sci., 60 (2012), 84-91.

[7] Allioglu, H., N. B. Bektas, M. Sayer. Stress Analysis of Functionally Graded Rotating Discs: Analytical and Numerical Solutions. Acta. Mech. Sin., 27 (2011), No. 6, 950-955.

[8] Ootao, Y., M. Ishinara. Transient Thermal Stress Problem of a Functionally Graded Magneto-electro-thermoelastic Hollow Sphere. Materials, 4 (2011), 21362150 .

[9] Arefi, M., G. H. Rahimi. The Effect of Nonhomogeneity and end Supports on the Thermo Elastic Behavior of a Clamped-clamped FG Cylinder under Mechanical and Thermal Loads. Int. J. Pres. Ves. Piping, 96-97 (2012), 30-37.

[10] Rahimi, G. H., M. Arefi, M. J. Khoshgoftar. Application and Analysis of Functionally Graded Piezoelectrical Rotating Cylinder as Mechanical Sensor Subjected to Pressure and Thermal Loads. Appl. Math. Mech. (Engl. Edition), 32 (2011), No. 8, 1-12.

[11] Arefi, M. Nonlinear Thermoelastic Analysis of Thick-walled Functionally Graded Piezoelectric Cylinder. Acta. Mech., 224 (2013), 2771-2783.

[12] Arefi, M., G. H. Rahimi. Comprehensive Thermoelastic Analysis of a Functionally Graded Cylinder with Different Boundary Conditions under Internal Pressure using First Order Shear Deformation Theory. Mechanika, 18 (2012), No. 1, 5-13.

[13] Arefi, M., G. H. Rahimi, M. J. Khoshgoftar. Electro Elastic Analysis of a Pressurized Thick-walled Functionally Graded Piezoelectric Cylinder using the First Order Shear Deformation Theory and Energy Method. Mechanika, 18 (2012), No. 3, 292-300.

[14] Arefi, M., G. H. Rahimi. Thermo Elastic Analysis of a Functionally Graded Cylinder under Internal Pressure using First Order Shear Deformation Theory. Sci. Res. Essays, 5 (2010), No. 12, 1442-1454. 
[15] Arefi, M., G. H. Rahimi, M. J. Khoshgoftar. Optimized Design of a Cylinder under Mechanical, Magnetic and Thermal Loads as a Sensor or Actuator using a Functionally Graded Piezomagnetic Material. Int. J. Phys. Sci., 6 (2011), No. 27, 6315-6322. 